\title{
ADDITIVE COMBINATIONS OF SPECIAL OPERATORS
}

\author{
PEI YUAN WU \\ Department of Applied Mathematics, National Chiao Tung University \\ Hsinchu, Taiwan 30050, Republic of China
}

\begin{abstract}
This is a survey paper on additive combinations of certain special-type operators on a Hilbert space. We consider (finite) linear combinations, sums, convex combinations and/or averages of operators from the classes of diagonal operators, unitary operators, isometries, projections, symmetries, idempotents, square-zero operators, nilpotent operators, quasinilpotent operators, involutions, commutators, self-commutators, norm-attaining operators, numerical-radius-attaining operators, irreducible operators and cyclic operators. In each case, we are mainly concerned with the characterization of such combinations and the minimal number of the special operators required in them. We will omit the proofs of most of the results here but give some indication or brief sketch of the ideas behind and point out the remaining open problems.
\end{abstract}

1. Introduction. In the study of operators on Hilbert spaces, the Cartesian decomposition is a fundamental and useful tool. In this case, we express an operator $T$ as an additive combination of two Hermitian operators: $T=T_{1}+i T_{2}$, where $T_{1}$ and $T_{2}$ are the real and imaginary parts of $T$. In operator theory, there are many other results of a similar nature: certain operators can be expressed as an additive combination of special-type operators. The aim of such decompositions is quite obvious. We try to gain insight into the structure of operators in the former class through our understanding of operators in the latter. Results of this nature are interesting by themselves and they often open a new vista of the operator terrain. The purpose of this paper is to survey such results, indicate their applications and point out the remaining open problems.

1991 Mathematics Subject Classification: Primary 47A05.

The paper is in final form and no version of it will be published elsewhere.

The author is very grateful to Drs. W. Żelazko and J. Zemánek for inviting him to take part in the Banach Semester activities and the hospitality extended to him during his stay in Warsaw. This survey paper covers some of the topics lectured upon during that time, and is partially supported by the National Science Council of Taiwan, China. 
Throughout this paper, we consider only bounded linear operators on a complex separable (finite- or infinite-dimensional) Hilbert space. (Some of the results here are also true for nonseparable Hilbert spaces. We restrict ourselves to the separable ones for ease of exposition.) Let $H$ be such a space and let $\mathcal{B}(H)$ denote the algebra of all operators on $H$. For a class of operators $\mathcal{S} \subseteq \mathcal{B}(H)$, we may consider different types of additive combinations. For one thing, we have linear combinations of (finitely many) elements of $\mathcal{S}$ :

$$
\lambda_{1} T_{1}+\ldots+\lambda_{n} T_{n},
$$

where the $\lambda_{j}$ 's are scalars. We may impose certain constraints on the coefficients $\lambda_{j}$ : if they are all equal to 1 , we have finite sums of elements of $\mathcal{S}$; if they are positive $\left(\lambda_{j} \geq 0\right)$, we have positive linear combinations. In some cases, we also consider convex combinations $\left(\lambda_{j} \geq 0\right.$ and $\left.\sum_{j} \lambda_{j}=1\right)$ and averages $\left(\lambda_{j}=1 / n\right)$. For each class $\mathcal{S}$ of operators, the problems to be asked are:

(1) how to characterize operators which are expressible as such combinations of elements from $\mathcal{S}$,

(2) what the smallest integer $n$ is for which every such operator can be written as a combination of $n$ elements from $\mathcal{S}$, and

(3) how to characterize, for each $m, 2 \leq m \leq n$, operators expressible as combinations of $m$ elements from $\mathcal{S}$.

We start, in Section 2 below, with additive combinations of diagonal operators. Here the recent work of Fong and the author [19] will be briefly surveyed. Then we move onto unitary operators and projections in Sections 3 and 4 . The additive combination problems for these two classes of operators have been studied more extensively. In the former case, the theory, in the context of von Neumann and $C^{*}$-algebras, culminates in the complete characterization of convex combinations (averages) of unitary operators and the determination of the minimal number of unitaries in such expressions $[36,42,20]$. In the latter, the attack on such problems has been successful only on finite-dimensional spaces [6]. Section 5 discusses combinations of some quadratic operators including idempotents, square-zero operators and involutions, and the related nilpotent and quasinilpotent operators. We conclude in Section 6 with combinations of operators from miscellaneous classes: commutators, self-commutators, norm-attaining operators, numerical-radius-attaining operators, irreducible operators and cyclic operators. They share the common property that every operator on $H$ is the sum of two such operators (except for self-commutators, in which case every Hermitian operator is).

Due to the expository nature of this paper, we will omit the proofs of most of the results here and refer the reader to the original paper. From time to time, we try to give the flavor and the main idea behind the scene. Along the way, we will also indicate the remaining open problems and unexplored areas. 
2. Diagonal operator. An operator is diagonal if it is unitarily equivalent to an operator of the form

$$
\left[\begin{array}{cccc}
a_{1} & & & \\
& a_{2} & 0 & \\
& & a_{3} & \\
0 & & \ddots
\end{array}\right]
$$

On a finite-dimensional space, every operator is the sum of two diagonal operators. This is because of the Cartesian decomposition and the fact that on a finitedimensional space every normal operator and, a priori, every Hermitian operator is diagonal. Hence for the rest of the section, we confine ourselves to operators on infinite-dimensional spaces. Our problem is: which operator is the sum of (finitely many) diagonal operators? The not too surprising answer is that every operator is. Here is the reason.

First note that every compact operator is the sum of two diagonal operators. This follows from the fact that the real and imaginary parts of a compact operator are also compact and that compact normal operators are diagonal (cf. [24, Corollary to Problem 172]). This suggests that the Weyl-von Neumann-Berg theorem on compact perturbations [2] may play a role. As it turns out, this is indeed the case. Using this theorem, we can prove the following

Proposition 2.1. (1) Every Hermitian operator is the sum of two diagonal operators.

(2) Every normal operator is the sum of three diagonal operators.

(3) Every operator is the sum of four diagonal operators.

So, the next problem is: can we do better? Or, in other words, can every operator be expressed as the sum of three or fewer diagonal operators? It turns out that "three" is the smallest such number. This is proved in [19] using the (block) tridiagonal representation of operators.

Recall that an operator is tridiagonal if it is unitarily equivalent to the direct sum of operators of the form

$$
\left[\begin{array}{cccc}
a_{0} & b_{0} & & \\
c_{0} & a_{1} & b_{1} & \\
& c_{1} & a_{2} & \ddots \\
& & \ddots & \ddots
\end{array}\right] \text { on } \ell^{2} \quad \text { or }\left[\begin{array}{llll}
a_{0} & b_{0} & & \\
c_{0} & & \ddots & \\
& \ddots & \ddots & b_{n-2} \\
& & c_{n-2} & a_{n-1}
\end{array}\right] \text { on } \mathbb{C}^{n}
$$

$(n \geq 1)$, where the unspecified entries are all zero; it is block tridiagonal if it is unitarily equivalent to the direct sum of 


$$
\left[\begin{array}{cccc}
A_{0} & B_{0} & & \\
C_{0} & A_{1} & B_{1} & \\
& C_{1} & A_{2} & \ddots \\
& & \ddots & \ddots
\end{array}\right] \quad \text { on } K_{0} \oplus K_{1} \oplus \ldots
$$

where the $K_{j}$ 's are all finite-dimensional. The folk theorem that every Hermitian operator is tridiagonal can be used to give an alternative proof of Proposition 2.1(1) (cf. [19, Corollary 3.2]). Indeed, if $T$ is Hermitian and of the form

then

$$
\left[\begin{array}{cccc}
a_{0} & b_{0} & & \\
c_{0} & a_{1} & b_{1} & \\
& c_{1} & a_{2} & \ddots \\
& & \ddots & \ddots
\end{array}\right]
$$

$$
T=\left[\begin{array}{ccccc}
a_{0} & b_{0} & & & \\
c_{0} & a_{1} & & & \\
& & a_{2} & b_{2} & \\
& & c_{2} & a_{3} & \\
& & & & \ddots
\end{array}\right]+\left[\begin{array}{cccccc}
0 & & & & & \\
& 0 & b_{1} & & & \\
& c_{1} & 0 & & & \\
& & 0 & b_{3} & \\
& & c_{3} & 0 & \\
& & & & \ddots
\end{array}\right] .
$$

It is easy to see that every $2 \times 2$ block appearing above is also Hermitian whence diagonal. Hence the above expression yields a sum of two diagonal operators for $T$. On the other hand, it can be shown that every operator has a block tridiagonal representation (cf. [19, Lemma 2.2]). A slightly more elaborate argument than above may prove the following theorem [19, Theorem 3.3]:

ThEOREM 2.2. Every operator is the sum of three diagonal operators.

The question then arises as to whether every operator is the sum of two diagonal operators. The answer turns out to be NO: the simple unilateral shift cannot be written as the sum of two diagonal operators. In fact, more can be proved: no nonnormal hyponormal operator is the sum of a diagonal operator and a normal operator (cf. [19, Corollary 3.5]). Here is the proof. If $T$ is a hyponormal operator such that $T=T_{1}+T_{2}$, where $T_{1}$ is diagonal $\left(T_{1} e_{n}=\lambda_{n} e_{n}\right.$ for the orthonormal basis $\left.\left\{e_{n}\right\}\right)$ and $T_{2}$ is normal, then a simple computation shows that $\left\langle\left(T^{*} T-T T^{*}\right) e_{n}, e_{n}\right\rangle=0$ for all $n$. Since $T^{*} T \geq T T^{*}$, we infer that $\left(T^{*} T-\right.$ $\left.T T^{*}\right) e_{n}=0$. Hence $T^{*} T=T T^{*}$, that is, $T$ is normal. More general results along this line may be obtained by an elaboration of such arguments. So for diagonal operators, we have a rather satisfactory situation.

We conclude this section with two related questions: (1) Is there a simple characterization of sums of two diagonal operators? (2) Is every normal operator the sum of two diagonal operators? Some partial results and pertinent comments can be found in [19]. 
3. Unitary operator. Which operator is the linear combination of unitary ones? A well-known result says that every operator is and the number of unitary operators needed is at most four. A mild modification of its usual proof yields a much stronger conclusion [36, Theorem 3.4]:

THEOREM 3.1. Every contraction is the sum of three unitary operators.

Indeed, if $T$ is a contraction $(\|T\| \leq 1)$, then in its Cartesian decomposition $T=T_{1}+i T_{2}$, the real and imaginary parts $T_{1}$ and $T_{2}$ are also contractions. Let

$$
A=\frac{1}{2}\left(T_{1}-\left(I-T_{1}^{2}\right)^{1 / 2}\right)
$$

and note that $A$ is a contraction. Thus we may define the unitary operators

$$
U=A+i\left(I-A^{2}\right)^{1 / 2} \text { and } V=\left(I-T_{2}^{2}\right)^{1 / 2}+i T_{2} .
$$

It is easily seen that $T=U+U^{*}+V$.

The following corollaries then follow immediately.

COROLlary 3.2. (1) Every operator is a positive multiple of the sum of three unitary operators.

(2) Every operator $T$ is the sum of at most $3 n$ unitary operators, where $n$ is the smallest integer no less than $\|T\|$.

For two unitary operators, we have

Proposition 3.3. (1) $T$ is a linear combination of two unitary operators if and only if $\operatorname{dim} \operatorname{ker} T=\operatorname{dim} \operatorname{ker} T^{*}$.

(2) $T$ is an average of two unitary operators if and only if $T$ is a contraction and $\operatorname{dim} \operatorname{ker} T=\operatorname{dim} \operatorname{ker} T^{*}$.

(3) $T$ is a linear combination of two commuting unitary operators if and only if $T$ is normal.

(4) $T$ is an average of two commuting unitary operators if and only if $T$ is a normal contraction.

We prove (2) and leave the other assertions to the reader. If $T=\frac{1}{2}(U+V)$, where $U$ and $V$ are unitary, then obviously $T$ is a contraction and

$$
\begin{aligned}
\operatorname{dim} \operatorname{ker} T & =\operatorname{dim} \operatorname{ker}\left(2 U^{*} T\right)=\operatorname{dim} \operatorname{ker}\left(I+U^{*} V\right)=\operatorname{dim} \operatorname{ker}\left(I+V^{*} U\right) \\
& =\operatorname{dim} \operatorname{ker}\left(2 T^{*} U\right)=\operatorname{dim} \operatorname{ker} T^{*},
\end{aligned}
$$

where the middle equality follows from the fact that a contraction and its adjoint have the same fixed points (cf. [41, p. 408]). Conversely, if $T$ is a contraction with $\operatorname{dim} \operatorname{ker} T=\operatorname{dim} \operatorname{ker} T^{*}$, then $T$ has the polar decomposition $T=U P$, where $U$ is unitary and $P=\left(T^{*} T\right)^{1 / 2}$ is a positive contraction. Since $P$ is the average of the two unitary operators

$$
P \pm i\left(I-P^{2}\right)^{1 / 2}
$$

$T$ is the average of the unitary

$$
U\left(P \pm i\left(I-P^{2}\right)^{1 / 2}\right) .
$$


These results appeared frequently in the literature (cf. [28, Theorem 3], [36, Lemma 2.1] and [27, Lemma]).

We next turn to the problems of convex combinations and averages (of unitary operators). The study of such problems has been very active in recent years. They are now completely solved in the contexts of von Neumann algebras [36] and $C^{*}$ algebras $[42,20]$. In the following, we briefly sketch these results for the present situation.

First note that for our purpose there is no need to distinguish between convex combinations and averages since it is shown in [29, Corollary 15] that every convex combination of unitary operators is the average of the same number of (possibly different) unitary ones. The proof is based on a careful analysis of the permissible coefficients in the expression of a Hermitian contraction as a convex combination of two unitary operators. This allows each convex combination of unitary operators to be expressed as every other such combination (with the same number of unitary operators) for which the coefficients are "closer to the average". The asserted result then follows.

For any operator $T$, let $u(T)$ denote the smallest integer for which $T$ is an average of $u(T)$ many unitary operators; $u(T)=\infty$ if there is no such representation for $T$. It turns out that the value of $u(T)$ depends on the distance $\alpha(T)$ from $T$ to the class of invertible operators. Recall that

$$
\alpha(T)= \begin{cases}0 & \text { if } \operatorname{dim} \operatorname{ker} T=\operatorname{dim} \operatorname{ker} T^{*}, \\ \max \left\{m_{\mathrm{e}}(T), m_{\mathrm{e}}\left(T^{*}\right)\right\} & \text { otherwise, }\end{cases}
$$

where $m_{\mathrm{e}}(T)=\inf \left\{\lambda: \lambda \in \sigma_{\mathrm{e}}\left(\left(T^{*} T\right)^{1 / 2}\right)\right\}$ is the essential minimum modulus of $T$ (cf. [4, Theorem 3]). Note that $0 \leq \alpha(T) \leq\|T\|$ for any operator $T$. The following theorem in [36] determines $u(T)$ completely.

THEOREM 3.4. If $T$ is a contraction, then

(1) $u(T) \leq 2$ if $\operatorname{dim} \operatorname{ker} T=\operatorname{dim} \operatorname{ker} T^{*}$,

(2) $u(T)=3$ if $\operatorname{dim} \operatorname{ker} T \neq \operatorname{dim} \operatorname{ker} T^{*}$ and $\alpha(T)=0$,

(3) $u(T)=n$ if

$$
1-\frac{2}{n-1}<\alpha(T) \leq 1-\frac{2}{n} \quad(n \geq 3),
$$

(4) $u(T)=\infty$ if $\alpha(T)=1$.

It may seem curious why the distance $\alpha(T)$ should get involved here. The fact is that it enters the picture through another parameter of $T$ : the distance from $T$ to the class of unitary operators. We recall that this distance is equal to

$$
\max \{\|T\|-1,1-m(T)\}
$$

if $\operatorname{dim} \operatorname{ker} T=\operatorname{dim} \operatorname{ker} T^{*}$, and

$$
\max \{\|T\|-1,1+\alpha(T)\}
$$


otherwise (cf. [35]). Here $m(T)=\inf \left\{\lambda: \lambda \in \sigma\left(\left(T^{*} T\right)^{1 / 2}\right)\right\}$ is the minimum modulus of $T$. To give some flavor of the proof of the preceding theorem, we treat a very special case of (3) above by showing that $T$ is the average of $n$ unitary operators if $\|T\|<1-2 / n(n \geq 3)$ (this is the case first proved in [29]). Indeed, let $S=\frac{1}{n-1}(n T-I)$ and $U$ be any unitary operator. Since

$$
\left\|\frac{1}{2}(U+S)\right\|=\frac{1}{2}\left\|I+U^{*} S\right\| \leq \frac{1}{2}(1+\|S\|)<1
$$

and, by [41, p. 408],

$\operatorname{dim} \operatorname{ker} \frac{1}{2}(U+S)=\operatorname{dim} \operatorname{ker}\left(I+U^{*} S\right)=\operatorname{dim} \operatorname{ker}\left(I+S^{*} U\right)=\operatorname{dim} \operatorname{ker} \frac{1}{2}\left(U^{*}+S^{*}\right)$,

Proposition 3.3(2) implies that $U+S$ is the sum of two unitary operators. Applying this observation inductively to $I+(n-1) S$ yields the asserted expression for $T$.

As proved above, every strict contraction $(\|T\|<1)$ is an average of unitary operators. This fact, in the context of Banach algebras with a * operation, can be used to prove the redundancy of the condition that the ${ }^{*}$ operation is isometric among the original six conditions proposed by Gelfand and Naimark for a $C^{*}$ algebra (cf. [29, Section 6]).

An easy consequence of the above theorem is a complete characterization of convex combinations (averages) of unitary operators [36, Proposition 2.12]:

Proposition 3.5. $T$ is a convex combination (average) of unitary operators if and only if it is a contraction not of the form $V+K$, where $V$ is a nonunitary isometry or coisometry and $K$ is compact.

We end this section with a few words about isometries. As with unitary operators, we may ask corresponding questions concerning their linear combinations, sums, convex combinations and averages. There are very few discussions about them in the literature. The following is a result which can be proved using arguments similar to those for Proposition 3.3.

Proposition 3.6. (1) $T$ is a linear combination of two isometries if and only if $\operatorname{dim} \operatorname{ker} T \leq \operatorname{dim} \operatorname{ker} T^{*}$.

(2) $T$ is an average of two isometries if and only if $T$ is a contraction and $\operatorname{dim} \operatorname{ker} T \leq \operatorname{dim} \operatorname{ker} T^{*}$.

Note that, using the polar decomposition, we can show that every contraction is the average of two operators, one of which is an isometry and the other a coisometry (cf. [24, Solution 136]). This is in contrast with the above assertions.

4. Projection. By projection we mean a Hermitian idempotent $\left(T^{*}=T\right.$ $=T^{2}$ ). The study of their linear combinations has a long history, dating back at least thirty-five years. It is Davis who first gave a complete characterization of the difference of two projections [8, Theorem 6.1]: 
Proposition 4.1. $T$ is the difference of two projections if and only if it is unitarily equivalent to an operator of the form $A \oplus I \oplus(-I)$, where $A$ satisfies $-1 \leq A \leq 1$ and is unitarily equivalent to $-A$.

We will provide a proof below based on a model for two projections.

If $P$ and $Q$ are any two projections on the space $H$, then we can decompose $H$ as the direct sum of five mutually orthogonal subspaces:

$$
\text { ran } P \cap \operatorname{ran} Q, \operatorname{ran} P \cap \operatorname{ran} Q^{\perp}, \operatorname{ran} P^{\perp} \cap \operatorname{ran} Q, \operatorname{ran} P^{\perp} \cap \operatorname{ran} Q^{\perp}
$$

and the orthogonal complement of the span of the preceding four subspaces. With respect to this decomposition, $P$ and $Q$ can be represented as

$$
I \oplus I \oplus 0 \oplus 0 \oplus P_{1} \quad \text { and } \quad I \oplus 0 \oplus I \oplus 0 \oplus Q_{1},
$$

respectively. The projections $P_{1}$ and $Q_{1}$ satisfy

$$
\begin{aligned}
\operatorname{ran} P_{1} \cap \operatorname{ran} Q_{1} & =\operatorname{ran} P_{1} \cap \operatorname{ran} Q_{1}^{\perp}=\operatorname{ran} P_{1}^{\perp} \cap \operatorname{ran} Q_{1} \\
& =\operatorname{ran} P_{1}^{\perp} \cap \operatorname{ran} Q_{1}^{\perp}=\{0\} .
\end{aligned}
$$

Exploiting the trigonometric analogy with two lines on the plane, we may show that the generic $P_{1}$ and $Q_{1}$ are simultaneously unitarily equivalent to projections of the form

$\left[\begin{array}{cc}A & (A(I-A))^{1 / 2} \\ (A(I-A))^{1 / 2} & I-A\end{array}\right]$ and $\left[\begin{array}{cc}A & -(A(I-A))^{1 / 2} \\ -(A(I-A))^{1 / 2} & I-A\end{array}\right]$, where $0 \leq A \leq 1$ (cf. [8, Theorem 6.2] or [23, p. 389]). The necessity of the preceding proposition then follows immediately. For the converse, if $A$ satisfies $-1 \leq A \leq 1$ and is unitarily equivalent to $-A$, then, by the spectral theorem for normal operators, $A$ is unitarily equivalent to an operator of the form $B \oplus(-B) \oplus 0$ with $0<B \leq 1$. Let

$P=\frac{1}{2}\left[\begin{array}{cc}I+B & \left(I-B^{2}\right)^{1 / 2} \\ \left(I-B^{2}\right)^{1 / 2} & I-B\end{array}\right] \quad$ and $\quad Q=\frac{1}{2}\left[\begin{array}{cc}I-B & \left(I-B^{2}\right)^{1 / 2} \\ \left(I-B^{2}\right)^{1 / 2} & I+B\end{array}\right]$.

Then $P$ and $Q$ are projections and $P-Q=B \oplus(-B)$. Thus $A$, together with $T$, is the difference of two projections.

Note that an operator $T$ is the difference of projections $P$ and $Q$ if and only if $T+I$ is the sum of projections $P$ and $I-Q$. Thus we obtain the following corollary immediately:

COROLlary 4.2. $T$ is the sum (resp. average) of two projections if and only if it is unitarily equivalent to $A \oplus 0 \oplus 2$ (resp. $A \oplus 0 \oplus I)$, where $A$ satisfies $0 \leq A \leq 2$ (resp. $0 \leq A \leq 1)$ and is unitarily equivalent to $2-A$ (resp. $I-A)$.

In $[13$, p. 151], this result is proved directly. More generally, Nishio obtained in [34] a complete characterization of any real linear combination of two projections.

The problem of linear combinations of more projections has been studied by many people $[38,37,32,33,16]$. It is long known that every operator is a linear combination of a fixed number of projections. The difficulty is to find the 
smallest such number. At present, the number is known to be no bigger than 8 or 10 depending on whether the underlying space is finite- or infinite-dimensional.

THEOREM 4.3. On a finite-dimensional space, every Hermitian operator is a real linear combination of four projections and every operator is a linear combination of eight projections.

This is proved in [33] through some clever manipulations with linear combinations of two projections on a two-dimensional space. For each $n \geq 1$, let $k(n)$ denote the minimal integer for which every Hermitian operator on an $n$-dimensional space can be represented as a linear combination of $k(n)$ projections. By Theorem 4.3 , we have $k(n) \leq 4$. It is also proved in [33] that

$$
k(1)=1, \quad k(2)=k(3)=2 \quad \text { and } \quad k(4)=k(5)=k(6)=k(7)=3 .
$$

The remaining problem is to determine whether $k(n)=4$ for $n \geq 8$.

Turning to infinite-dimensional spaces, we have

THEOREM 4.4. On an infinite-dimensional space, every Hermitian operator is a real linear combination of five projections and every operator is a linear combination of ten projections.

This is contained in [32], together with the result that every Hermitian operator (on an infinite-dimensional space) can be expressed as an integral linear combination of six projections.

A projection $P$ is proper if $\operatorname{rank} P=\operatorname{rank} P^{\perp}=\infty$. Since every projection is either the sum or the difference of two proper projections [11, Lemma 2], we obtain immediately

COROLlARY 4.5. On an infinite-dimensional space, every operator is a linear combination of at most twenty proper projections.

The next question is about positive linear combinations of projections. We may wonder whether every positive operator has such a representation. The answer turns out to be NO. Indeed, as was observed in [11, p. 287], any positive infiniterank compact operator cannot be expressed in this way. Reason: If $T$ is such an operator and is a positive linear combination of projections, $T=\sum_{j=1}^{n} \lambda_{j} P_{j}$, then, since $0 \leq \lambda_{j} P_{j} \leq T$, the compactness of $T$ implies that of each $P_{j}$. Hence the rank of $P_{j}$ must be finite and the same is true for $T$. On the other hand, it is proved in [13] that every positive invertible operator is a positive linear combination of projections. The surprising thing is that the condition of infiniterank compactness forms the only obstruction for such representations.

THEOREM 4.6. On an infinite-dimensional space, $T$ is a positive linear combination of projections if and only if $T \geq 0$ and $T$ is not an infinite-rank compact operator.

The sufficiency of this theorem can be proved via the corresponding results for averages of projections [16, Theorem 3 and Lemma 9]. 
We next consider the related problems of convex combinations, averages and sums of projections. In the finite-dimensional case, a thorough study of the first two problems was conducted in [6]. Note that if $T$ is a convex combination of projections, then $T$ satisfies $0 \leq T \leq 1$. On a finite-dimensional space, the converse is also true. Thus, for example, if $T, 0 \leq T \leq 1$, is acting on a 3 -dimensional space, then, assuming that it is of the form

$$
\left[\begin{array}{lll}
a & 0 & 0 \\
0 & b & 0 \\
0 & 0 & c
\end{array}\right]
$$

with $0 \leq a \leq b \leq c \leq 1, T$ can be expressed as

$a\left[\begin{array}{lll}1 & 0 & 0 \\ 0 & 1 & 0 \\ 0 & 0 & 1\end{array}\right]+(b-a)\left[\begin{array}{lll}0 & 0 & 0 \\ 0 & 1 & 0 \\ 0 & 0 & 1\end{array}\right]+(c-b)\left[\begin{array}{lll}0 & 0 & 0 \\ 0 & 0 & 0 \\ 0 & 0 & 1\end{array}\right]+(1-c)\left[\begin{array}{ccc}0 & 0 & 0 \\ 0 & 0 & 0 \\ 0 & 0 & 0\end{array}\right]$,

a convex combination of four projections. Following a similar procedure, we can show that every $T, 0 \leq T \leq 1$, on an $n$-dimensional space can be written as a convex combination of $n+1$ projections. Note that in the above expression, the projections involved are mutually commuting. For such projections, the above expression is indeed optimal:

THEOREM 4.7. On an $n$-dimensional space, every operator, $T, 0 \leq T \leq 1$, is the convex combination of $n+1$ commuting projections. For each $n \geq 1$, the number $n+1$ is sharp.

In general, if we allow noncommuting projections in the combination, then the number should be fewer than $n+1$. This is indeed the case, as we now proceed to show that, in the previous example, the matrix $T$ can be expressed as a convex combination of three projections. The proof is based on the observation that for $0 \leq a \leq b \leq c$, there are rank-one projections $P$ and $Q$ such that

$$
\left[\begin{array}{ll}
a & 0 \\
0 & c
\end{array}\right]=b P+(a+c-b) Q
$$

(cf. [33, Lemma 2]). If $a+c \leq 1$, then

$$
\left[\begin{array}{lll}
a & 0 & 0 \\
0 & c & 0 \\
0 & 0 & b
\end{array}\right]=b\left[\begin{array}{cc}
P & 0 \\
0 & 1
\end{array}\right]+(a+c-b)\left[\begin{array}{cc}
Q & 0 \\
0 & 0
\end{array}\right]+(1-a-c)\left[\begin{array}{ll}
0 & 0 \\
0 & 0
\end{array}\right]
$$

is a convex combination of three projections, which proves our assertion. For the rest of the proof, we assume that $a+c>1$. We may also assume that $b \leq 1 / 2$ since otherwise we can consider $I-T$ instead. These assumptions imply that $0 \leq 1-c \leq b \leq 1-a$. Hence the preceding observation yields that

$$
\left[\begin{array}{cc}
1-a & 0 \\
0 & 1-c
\end{array}\right]=b P+(2-a-b-c) Q
$$


for some rank-one projections $P$ and $Q$. We then have

$$
\left[\begin{array}{ll}
a & 0 \\
0 & c
\end{array}\right]=b(I-P)+(1-b)\left(I-\frac{2-a-b-c}{1-b} Q\right)
$$

whence

$$
\left[\begin{array}{lll}
a & 0 & 0 \\
0 & c & 0 \\
0 & 0 & b
\end{array}\right]=b\left[\begin{array}{cc}
I-P & 0 \\
0 & 1
\end{array}\right]+(1-b)\left[\begin{array}{cc}
I-\frac{2-a-b-c}{1-b} Q & 0 \\
0 & 0
\end{array}\right] .
$$

It is easy to see that the last matrix, call it $A$, is of rank one and satisfies $0 \leq$ $A \leq 1$. Hence, by Theorem 4.7, it is a convex combination of two projections: $A=d R+(1-d) S$ with $0 \leq d \leq 1$ and projections $R$ and $S$. We conclude that

$$
\left[\begin{array}{lll}
a & 0 & 0 \\
0 & c & 0 \\
0 & 0 & b
\end{array}\right]=b\left[\begin{array}{cc}
I-P & 0 \\
0 & 0
\end{array}\right]+(1-b) d R+(1-b)(1-d) S
$$

is a convex combination of three projections.

Will two projections be enough? The answer is NO: the operator

$$
T=\left[\begin{array}{lll}
a & 0 & 0 \\
0 & b & 0 \\
0 & 0 & c
\end{array}\right]
$$

with $0 \leq a<b<c<a+b+c<1$ cannot be represented as the convex combination of two projections. Indeed, if $T=\lambda P+(1-\lambda) Q$, where $0 \leq \lambda \leq 1$ and $P$ and $Q$ are projections with $\operatorname{rank} P \leq \operatorname{rank} Q$, then

$1>a+b+c=\operatorname{tr} T=\lambda \operatorname{tr} P+(1-\lambda) \operatorname{tr} Q=\lambda \operatorname{rank} P+(1-\lambda) \operatorname{rank} Q \geq \operatorname{rank} P$ implies that $P=0$ and thus $T=(1-\lambda) Q$, which is certainly impossible.

A more refined argument than above can be used to prove the following

THEOREM 4.8. On an n-dimensional space, every operator $T, 0 \leq T \leq 1$ (resp. $T \geq 0$ ), is a convex combination (resp. positive linear combination) of $\left[\log _{2} n\right]+2$ (resp. $\left.\left[\log _{2} n\right]+1\right)$ projections. For each $n \geq 1$, this number is sharp.

Here for any real number $x,[x]$ denotes its integral part.

Note that the difference of the numbers of projections in the preceding theorem reflects the fact that convex combinations require one more constraint on the coefficients than positive linear combinations: their sum must be zero.

For averages of projections, we have the following theorem from [6, Theorem 3.6]: 
TheOREM 4.9. Let $T$ be an operator on an $n$-dimensional space.

(1) $T$ is an average of projections if and only if $0 \leq T \leq 1$ and $\operatorname{tr} T$ is rational.

(2) $T$ is an average of commuting projections if and only if $0 \leq T \leq 1$ and the eigenvalues of $T$ are all rational.

In both cases, the minimal number of projections required can be arbitrarily large for every fixed $n \geq 1$.

In [6], (1) is proved based on the representation of operators as averages of equal-rank projections. We give below an alternative and more direct proof connecting it with sums of projections [13, Theorem 1].

THEOREM 4.10. On a finite-dimensional space, $T$ is a sum of projections if and only if $T \geq 0, \operatorname{tr} T$ is an integer and $\operatorname{tr} T \geq \operatorname{rank} T$. In this case, $T$ can be written as the sum of at most $[\|T\|]+\operatorname{rank} T$ projections.

The necessity is obvious. For the sufficiency, assume that

$$
T=\left[\begin{array}{ccc}
a_{1} & & 0 \\
& \ddots & \\
0 & & a_{n}
\end{array}\right],
$$

$a_{1} \geq \ldots \geq a_{n}>0$, satisfies the stated conditions. If $\operatorname{tr} T>\operatorname{rank} T$, that is, $\sum_{j} a_{j}>n$, then $a_{j}>1$ for some $j$. Subtracting the projection

$$
j \text { th }\left[\begin{array}{ccccc}
0 & & j \text { th } & & \\
& \ddots & & 0 & \\
& & 1 & & \\
& 0 & & \ddots & \\
& & & & 0
\end{array}\right]
$$

from $T$ yields an operator with trace reduced by 1 . Repeating this process, we are reduced to the case $\operatorname{tr} T=\operatorname{rank} T=n$. The subtracted projections which are on different coordinate spaces can be pooled together, so that their total number is at most $[\|T\|]$. The proof then proceeds as in [13] so that operators satisfying this additional condition can be expressed as a sum of $n$ rank-one projections. Thus the total number of projections needed for $T$ is at most $[\|T\|]+\operatorname{rank} T$.

Returning to the proof of Theorem 4.9(1), assume that $T, 0 \leq T \leq 1$, acts on an $n$-dimensional space with $\operatorname{tr} T$ rational. It suffices to consider the case $0<T<1$. Write $\operatorname{tr} T=r / s$, where $r$ and $s$ are large positive integers with $r \geq \operatorname{rank} T$. Then $s T$ is an operator satisfying the conditions in Theorem 4.10. Hence $s T=\sum_{j=1}^{m} P_{j}$ is a sum of $m$ projections with $m \leq[s\|T\|]+\operatorname{rank} T$. Since $\|T\|<1$, for sufficiently large $s$ we have

$$
\frac{m}{s} \leq\|T\|+\frac{1}{s} \operatorname{rank} T<1
$$


or $m<s$. Therefore,

$$
T=\frac{1}{s}(P_{1}+\ldots+P_{m}+\underbrace{0+\ldots+0}_{s-m})
$$

is an average of $s$ projections.

As for the unboundedness of the number of projections in an average, this is already evident in the one-dimensional case: the $1 \times 1$ matrix $[1 / n]$ can only be written as an average of $n k$ projections for some positive integer $k$ ( $k$ of them are [1] and the rest [0]), whence an average of at least $n$ projections.

On infinite-dimensional spaces, the problems of characterizing convex combinations, averages and sums (of projections) have not been completely settled. Fong and Murphy [16] are the first to consider the convex combinations and averages. They obtained a number of conditions, either necessary or sufficient, for an operator in order that it be expressible as such. Among them is the following

THEOREM 4.11. Let $T, 0 \leq T \leq 1$, be an operator on an infinite-dimensional space such that its essential spectrum $\sigma_{\mathrm{e}}(T)$ intersects the unit open interval $(0,1)$. Then $T$ is an average of projections.

The proof is a tour de force through spectral properties of $T$.

To complete the characterization of convex combinations or averages of projections, it remains to determine which operator $T, 0 \leq T \leq 1$, with the properties that $\sigma_{\mathrm{e}}(T)=\{0,1\}$ and 0 and 1 are both limit points of $\sigma(T)$ has such a representation. This has been open for many years. Note that not every such operator can be expressed in this way. For example, it can be verified that if $T=K_{1} \oplus\left(I-K_{2}\right)$ is a convex combination of projections, where $K_{1}$ and $K_{2}$ are infinite-rank compact operators satisfying $0 \leq K_{1}, K_{2} \leq 1$, then $K_{1}$ is of trace class if and only if $K_{2}$ is.

Finally, we come to the characterization problem for sums of projections (on an infinite-dimensional space). Partial progress has been made in [13] and [7], but a complete solution seems difficult to obtain at the present time. From what we have so far, it seems that the structure of sums of projections is parallel to that of averages of projections. This is not too surprising considering the similarity of their respective expressions. One sufficient condition for sums of projections is analogous to Theorem 4.11 above:

THEOREM 4.12. Let $T, T \geq 0$, be an operator on an infinite-dimensional space such that $\sigma_{\mathrm{e}}(T)$ intersects the open interval $(1, \infty)$. Then $T$ is a sum of projections.

The proof is through some clever manipulations based on the observation that $T=T_{1} \oplus \ldots \oplus T_{n}$ on $H \oplus \ldots \oplus H$ with $T_{j} \geq 0$ for all $j$ and $T_{1}+\ldots+T_{n}=n I$ is a sum of projections.

As before, to obtain a complete characterization, we are left with one open case: if $T=I+K_{1}-K_{2}$ is injective, where $K_{1}$ and $K_{2}$ are positive infiniterank compact operators with $\operatorname{tr} K_{1}=\operatorname{tr} K_{2}=\infty$ or $\operatorname{tr} K_{1}-\operatorname{tr} K_{2}$ a nonnegative 
integer, is $T$ a sum of projections? We suspect that the answer is affirmative. Note that this result, if it is indeed true, is consistent with the characterization in the finite-dimensional case (Theorem 4.10): if $T>0$ is written as $I+K_{1}-K_{2}$ on a finite-dimensional space, where $K_{1}, K_{2} \geq 0$, then the condition for $T$ to be a sum of projections can be formulated as $\operatorname{tr} K_{1}-\operatorname{tr} K_{2}$ being a nonnegative integer.

A class of operators closely related to projections is that of symmetries $(T=$ $\left.T^{*}=T^{-1}\right)$. Since $P$ is a projection if and only if $2 P-I$ is a symmetry, these two classes are in one-to-one correspondence. Hence results in this section can be suitably transformed into ones for symmetries. In particular, we have

THEOREM 4.13. (1) $T$ is an average of two symmetries if and only if $T$ is unitarily equivalent to $A \oplus I \oplus(-I)$, where $A$ satisfies $-1 \leq A \leq 1$ and is unitarily equivalent to $-A$.

(2) On a finite-dimensional (resp. infinite-dimensional) space, every Hermitian operator is a real linear combination of five (resp. six) symmetries.

(3) On an infinite-dimensional space, every Hermitian operator is a sum of symmetries.

(1) of the preceding theorem appeared in [13, p. 150]; (3) follows from the fact that every Hermitian operator $T$ with $-2 \leq T \leq 2$ is a sum of eight symmetries, which is, in turn, deducible from (1) (cf. [13, Theorem 3]).

5. Quadratic operator. An operator $T$ is quadratic if $p(T)=0$ for some quadratic polynomial $p$. Since every such operator can be transformed, through a linear function, to an idempotent operator $\left(T^{2}=T\right)$ or a square-zero operator $\left(T^{2}=0\right)$, we start by considering linear combinations of idempotents.

Our first result gives a characterization of differences of two idempotents on a finite-dimensional space [25]:

THEOREM 5.1. On a finite-dimensional space, $T$ is a difference of two idempotents if and only if $T$ is similar to an operator of the form $A \oplus B \oplus(-B) \oplus$ $(I-C D) \oplus(D C-I)$, where $A, C D$ and $D C$ are all nilpotent and 0 and \pm 1 are not eigenvalues of $B$.

Recall that an operator $S$ is nilpotent if $S^{n}=0$ for some $n \geq 1$.

Generalizing this to infinite-dimensional spaces seems difficult. In [46] and [50], there are derived necessary or sufficient conditions for an operator to be expressible as such a difference, which can be considered as partial generalizations of the preceding theorem.

THEOREM 5.2. (1) If $T$ is unitarily equivalent to $-T$, then $T$ is a difference of two idempotents.

(2) If $T$ is a difference of two idempotents, then $\sigma(T) \backslash\{ \pm 1\}=\sigma(-T) \backslash\{ \pm 1\}$ and $\sigma_{\mathrm{e}}(T) \backslash\{ \pm 1\}=\sigma_{\mathrm{e}}(-T) \backslash\{ \pm 1\}$.

Here we only sketch briefly the proof of (2). If $T=P-Q$ is the difference of two idempotents, let $S=I-P-Q$. Since $(S+T)^{2}=(S-T)^{2}=I$, we have 
$\sigma(S+T), \sigma(S-T) \subseteq\{ \pm 1\}$. Hence for any complex number $\lambda \neq \pm 1, S+T-\lambda I$ and $S-T-\lambda I$ are invertible. From the identity

$$
(T-\lambda I)(S-T-\lambda I)=(S+T-\lambda I)(-T-\lambda I),
$$

we infer that $T-\lambda I$ is invertible (resp. Fredholm) if and only if $-T-\lambda I$ is. This proves our assertions in (2).

For normal operators, we do have a characterization, which is consistent with the conditions in Theorem 5.1 (cf. [46, Theorem 1.18] or [50, Theorem 1.19]).

TheOREM 5.3. A normal operator $T$ is a difference of two idempotents if and only if $T$ is unitarily equivalent to $A \oplus(-A) \oplus 0 \oplus I \oplus(-I)$, where $A$ is some normal operator such that 0 and \pm 1 are not its eigenvalues.

However, there are discrepancies between the finite- and the infinite-dimensional cases. The next result is proved in [50, Proposition 1.12].

THEOREM 5.4. (1) Every square-zero operator is a difference of two idempotents.

(2) There is an operator $T$ with $T^{3}=0$ which is not a difference of two idempotents.

As with projections, an operator $T$ is the difference of two idempotents if and only if $T+I$ is the sum of two idempotents. Hence the preceding results can be reformulated as conditions for the sum of two idempotents. We next consider sums of more idempotents. On a finite-dimensional space, such sums are characterized in [26] and [51].

TheOREM 5.5. On a finite-dimensional space, $T$ is a sum of idempotents if and only if $\operatorname{tr} T$ is an integer and $\operatorname{tr} T \geq \operatorname{rank} T$.

Note the similarity of these conditions with those for sums of projections in Theorem 4.10. As before, the necessity is obvious. To prove the sufficiency, we assume that $T$ is invertible on an $n$-dimensional space. If $T$ is nonscalar, then, since $\operatorname{tr} T \geq \operatorname{rank} T$, there are positive integers $t_{1}, \ldots, t_{n}$ such that $\sum_{j} t_{j}=\operatorname{tr} T$. By [12, Theorem 2], $T$ is similar to a matrix of the form

$$
\left[\begin{array}{lll}
t_{1} & & * \\
& \ddots & \\
* & & t_{n}
\end{array}\right] .
$$

This latter matrix can be decomposed as

$$
t_{1}\left[\begin{array}{cc}
1 & \\
* & \\
\vdots & 0 \\
* &
\end{array}\right]+t_{2}\left[\begin{array}{cc}
* & \\
1 & \\
0 & 0 \\
\vdots & \\
* &
\end{array}\right]+\ldots+t_{n}\left[\begin{array}{cc} 
& * \\
0 & \vdots \\
& * \\
& 1
\end{array}\right] .
$$


Since each of the above matrices is idempotent, we infer that $T$ is a sum of $\operatorname{tr} T\left(=\sum_{j} t_{j}\right)$ idempotents. On the other hand, if $T$ is a scalar operator, say, $T=a I(a \neq 0)$, then $\operatorname{tr} T \geq \operatorname{rank} T$ implies that $a \geq 1$. Thus

$$
T=\left[\begin{array}{cc}
1 & \\
0 & 0 \\
\vdots &
\end{array}\right]+\left[\begin{array}{cccc}
a-1 & & & 0 \\
& a & & \\
& & \ddots & \\
0 & & & a
\end{array}\right]
$$

the latter matrix being nonscalar with its trace not smaller than its rank. We conclude from the above that, again, $T$ is a sum of $\operatorname{tr} T$ idempotents.

In [51], it was shown that $\operatorname{tr} T$, the number of idempotents, is in general not optimal. For example, the $n \times n$ matrix

$$
\left[\begin{array}{cccc}
4 & & & 0 \\
& 0 & & \\
& & \ddots & \\
0 & & & 0
\end{array}\right]
$$

can be written as a sum of three idempotents. The problem of finding the minimal number of required idempotents is further pursued in [47].

In [26], there are also results concerning linear combinations of idempotents with integral or positive coefficients.

THEOREM 5.6. Let $T$ be an operator on a finite-dimensional space. Then $T$ is an integral (resp. positive) linear combination of idempotents if and only if $\operatorname{tr} T$ is an integer (resp. $T=0$ or $\operatorname{tr} T>0)$.

Such problems are recently further studied in [48].

THEOREM 5.7. Let $T$ be an operator on an $n$-dimensional space.

(1) $T$ is a linear combination of at most five idempotents.

(2) $T$ is a convex combination of idempotents if and only if $T=0, T=I$ or $0<\operatorname{tr} T<n$. In this case, five idempotents would suffice.

(3) $T$ is an average of idempotents if and only if $T=0, T=I$ or $0<\operatorname{tr} T<n$ and $\operatorname{tr} T$ is rational. In this case, the minimal number of idempotents required can be arbitrarily large for every fixed $n \geq 1$.

The problem of sums of idempotents on an infinite-dimensional space was first studied in [43]. Unfortunately, the main result of that paper that every Hermitian operator is the sum of four idempotents is false due to an error in its proof. A counterexample can be obtained from Proposition 5.9 below. The situation was rectified by Pearcy and Topping [38]. Using the characterization of commutators ( $T=A B-B A$ for some operators $A$ and $B$ if and only if $T$ is not of the form $\lambda I+K$, where $\lambda$ is a nonzero scalar and $K$ is compact [5]), they were able to prove 
THEOREM 5.8. (1) If $T$ is not of the form $\lambda I+K$, where $\lambda \neq 2$ is a scalar and $K$ is compact, then $T$ is a sum of four idempotents.

(2) Every operator is the sum of five idempotents.

So the problem remains as to whether every operator is a sum of four idempotents. That this is not the case can be seen from the next result.

Proposition 5.9. If $\lambda I+K$ is a sum of four idempotents, where $\lambda$ is a scalar and $K$ is compact, then either $\lambda=2$ or $\lambda=2 \pm 2 / n$ for some $n \geq 1$.

This is proved in [46, Corollary 1.20] using the symmetry property for the essential spectra of sums of two idempotents (cf. Theorem 5.2(2)). To conclude this part of the discussion, we propose the following

ConjeCture 5.10. An operator of the form $\lambda I+K$, where $\lambda$ is a scalar and $K$ is compact, is a sum of four idempotents if and only if $\lambda=2$ or $\lambda=2 \pm 2 / n$ for some $n \geq 1$.

As a supporting evidence, it is shown in [46] that the assertion is true if $K=0$. To give some idea of the proof for this case, we now proceed to show that $T=\frac{8}{3} I$ is a sum of four idempotents. Note that $T$ is unitarily equivalent to $\frac{8}{3} I \oplus \frac{8}{3} I \oplus \frac{8}{3} I$, which can be written as the sum,

$$
\left(\frac{2}{3} I \oplus \frac{4}{3} I \oplus \frac{6}{3} I\right)+\left(\frac{6}{3} I \oplus \frac{4}{3} I \oplus \frac{2}{3} I\right) .
$$

From Theorem 5.3, we infer that $\frac{2}{3} I \oplus \frac{4}{3} I$ is a sum of two idempotents. Hence $T$ is a sum of four idempotents as asserted.

We next consider another kind of quadratic operators: the square-zero ones. As expected, results for such operators are more or less parallel to those for idempotents. Most of the following can be found in [49].

On a finite-dimensional space, sums of two square-zero operators can be completely determined:

THEOREM 5.11. The following conditions are equivalent for an operator $T$ on a finite-dimensional space:

(1) $T$ is the sum of two square-zero operators;

(2) $T$ is similar to $-T$;

(3) $T$ is similar to an operator of the form $A \oplus B \oplus(-B)$, where $A$ is nilpotent and $B$ is invertible.

As with idempotents, we have only partial success in generalizing this to infinite-dimensional spaces.

TheOREM 5.12. (1) If $T$ is unitarily equivalent to $-T$, then $T$ is the sum of two square-zero operators.

(2) If $T$ is a sum of two square-zero operators, then $\sigma(T)=\sigma(-T)$ and $\sigma_{\mathrm{e}}(T)=\sigma_{\mathrm{e}}(-T)$. 
As before, the proof of (2) depends on the identity

$$
(T-\lambda I)(S-T-\lambda I)=(S+T-\lambda I)(-T-\lambda I),
$$

where $T=T_{1}+T_{2}$ is a sum of two square-zero operators and $S=T_{1}-T_{2}$.

For normal operators, we do have a characterization:

THEOREM 5.13. For a normal operator $T$, the following conditions are equivalent:

(1) $T$ is the sum of two square-zero operators;

(2) $T$ is unitarily equivalent to $-T$;

(3) $T$ is unitarily equivalent to an operator of the form $A \oplus(-A) \oplus 0$.

For sums of more square-zero operators, the following theorem gives their characterization on finite-dimensional spaces:

TheOREM 5.14. On a finite-dimensional space, $T$ is a sum of finitely many square-zero operators if and only if $\operatorname{tr} T=0$. In this case, four square-zero operators would suffice.

Again, the necessity is trivial. To prove the sufficiency, assume that $T$ has trace zero. Applying [12, Theorem 2], we find that $T$ is unitarily equivalent to a matrix of the form

$$
\left[\begin{array}{ccc}
0 & & * \\
& \ddots & \\
* & & 0
\end{array}\right]
$$

which can be written as the sum

$$
\left[\begin{array}{ccc}
0 & & * \\
& \ddots & \\
0 & & 0
\end{array}\right]+\left[\begin{array}{ccc}
0 & & 0 \\
& \ddots & \\
* & & 0
\end{array}\right] .
$$

Since each of these latter two matrices is nilpotent whence a sum of two squarezero operators by Theorem 5.11, our assertion follows immediately.

On the infinite-dimensional space, Pearcy and Topping [38] deduced the following result using the characterization of commutators:

THEOREM 5.15. (1) If $T$ is not of the form $\lambda I+K$, where $\lambda$ is a nonzero scalar and $K$ is compact, then $T$ is a sum of four square-zero operators.

(2) Every operator is the sum of five square-zero operators.

The situation for sums of four square-zero operators is easier to handle than for idempotents. The next theorem is the infinite-dimensional counterpart for Theorem 5.14 (cf. [49, Theorem 3.8]):

THEOREM 5.16. On an infinite-dimensional space, an operator is the sum of four square-zero operators if and only if it is a commutator.

In view of Theorem 5.15 and the characterization of commutators, we need only check that if the operator $\lambda I+K$, where $\lambda$ is a scalar and $K$ is compact, is 
a sum of four square-zero operators, then $\lambda$ must be zero. As with idempotents, this can be proved using the symmetry property of the essential spectra of sums of two square-zero operators (cf. Theorem 5.12(2)).

We remark that [11] also considered sums of square-zero operators and linear combinations of projections. However, the results therein are proved using the erroneous assertion in [43].

We next take a digression to the related classes of nilpotent operators $\left(T^{n}=0\right.$ for some $n \geq 1$ ) and quasinilpotent operators $(\sigma(T)=\{0\})$. Problems concerning their sums are studied in [18]. On a finite-dimensional space, the decomposition (*) yields

Proposition 5.17. On a finite-dimensional space, $T$ is a sum of nilpotent operators if and only if $\operatorname{tr} T=0$. In this case, two nilpotent operators would suffice.

On the other hand, for infinite-dimensional space, we have

THEOREM 5.18. On an infinite-dimensional space,

(1) an operator is a sum of two quasinilpotent operators if and only if it is a commutator, and

(2) every operator is the sum of three quasinilpotent operators.

(1) of the preceding theorem is proved in [18] via the construction of some Volterra-type integral operators. Note the contrast between the assertions in Theorem 5.18(1) and Theorem 5.16.

In-between the assertions of Theorems 5.15(2) and 5.18(2), there remains at least one open problem: since every operator is a sum of a fixed number of nilpotent operators, what is the minimal such number? In view of the previous theorems, the number should be between three and five.

There is another deep result in this area.

THEOREM 5.19. On an infinite-dimensional space, $T$ is a sum of quasinilpotent trace-class operators if and only if $T$ is of trace class with the eigenvalues $\left\{\lambda_{n}\right\}$, arranged so that $\left|\lambda_{n}\right|$ is decreasing, satisfying $\sum_{n} \frac{1}{n}\left|\lambda_{1}+\ldots+\lambda_{n}\right|<\infty$. In this case, $T$ is a sum of at most 24 such operators.

This is proved by Kalton [30, Theorem 7.4]; it is part of a chain of equivalent conditions on commutators involving trace-class operators. The paper [30] is innovative in that it introduces techniques which are an extension of ideas arising in interpolation theory.

Besides idempotents and square-zero operators, there is another class of quadratic operators which holds some mild interest. They are involutions $\left(T^{2}=I\right)$. Since they can be transformed to idempotents under the mapping $T \rightarrow \frac{1}{2}(T+I)$ and vice versa, their combinations can be obtained from the corresponding ones for idempotents. In particular, we have 
Proposition 5.20. (1) On a finite-dimensional space, $T$ is the sum of involutions if and only if $\operatorname{tr} T$ is an integer and, when the dimension of the underlying space is even, is even.

(2) On an infinite-dimensional space, every operator is the sum of five involutions.

(1) of the preceding proposition appeared in [51, Corollary 3].

6. Miscellany. In this last section, we consider combinations of operators from miscellaneous classes. The classes are all large enough so that we have the best situation we can hope for: every operator is the sum of two operators from such classes. In the end, we will give a general method, due to Fong et al. [15, 17], which is useful in obtaining linear combination results.

We start with an easy observation which does not seem to be widely noticed: every operator is the sum of two invertible operators. Reason: decompose every operator $T$ as $T=\lambda I+(T-\lambda I)$, where $\lambda$ is any nonzero scalar not in $\sigma(T)$.

The next class of operators we consider is that of commutators. As we have seen in Section 5, these operators are useful in solving problems concerning sums of certain quadratic operators. Now we will consider combinations of commutators. The next theorem says that their sums include all the operators.

THEOREM 6.1. On an infinite-dimensional space, every operator is the sum of two commutators.

This is first proved by Halmos [22]; it is an easy consequence of the fact that every operator with a large kernel is a commutator [24, Problem 234] ( $T$ on $H$ has a large kernel if $\operatorname{dim} \operatorname{ker} T=\operatorname{dim} H)$. One application of Theorem 6.1 is that there is no nonzero multiplicative linear functional on $\mathcal{B}(H)$ for any infinitedimensional $H$.

There is one longstanding open problem concerning commutators: is every compact operator the commutator of two compact operators? Anderson [1] showed that the answer is affirmative if we only require that one of the two operators for the commutator be compact. The next result provides another piece of supporting evidence for its validity.

THEOREM 6.2. On an infinite-dimensional space, every compact operator is the sum of two commutators each of which is formed by compact operators.

This is proved in $[10]$ based on the theory of zero-diagonal operators. It improves an earlier result with three such commutators [9].

Operators of the form $A^{*} A-A A^{*}$ are called self-commutators. They were first characterized by Radjavi [39]: on an infinite-dimensional space, $T$ is a selfcommutator if and only if $T$ is Hermitian and 0 belongs to the convex hull of 
the essential spectrum of $T\left(^{1}\right)$. Before this characterization was obtained, Halmos [21] was able to show the following result concerning their sums, basing it on the observation that every Hermitian operator with a large kernel is a selfcommutator.

THEOREM 6.3. On an infinite-dimensional space, every Hermitian operator is the sum of two self-commutators.

Using results on zero-diagonal operators, Fan and Fong [9] obtained the following analogue for compact operators:

THEOREM 6.4. On an infinite-dimensional space, every compact Hermitian operator is the sum of two compact self-commutators.

An operator $T$ is norm-attaining if there is a unit vector $x$ such that $\|T x\|=$ $\|T\|$. Using the compactness of the unit circle of a finite-dimensional space, it is easy to verify that every operator on such a space is norm-attaining. In the same spirit, we may show the same for every compact operator on an infinitedimensional space. Somewhat unexpected is the following

THEOREM 6.5. Every operator is a compact perturbation of a norm-attaining operator. Moreover, the norm of the compact operator can be made arbitrarily small.

We can draw two conclusions from the above result:

COROLlary 6.6. (1) Every operator is the sum of two norm-attaining operators.

(2) Norm-attaining operators on $H$ are $($ norm $)$ dense in $\mathcal{B}(H)$.

Theorem 6.5 was proved in [31] for operators from any reflexive Banach space to another Banach space. The proof entails the construction, for any operator $T$, of a sequence of operators $\left\{T_{n}\right\}$ such that $T-T_{n}$ is of finite rank for each $n$ and $T_{n}$ converges to some norm-attaining operator.

Parallel to the investigations here, [3] studied the corresponding problem for the numerical-radius-attaining operators. An operator $T$ is numerical-radiusattaining if there is a unit vector $x$ such that $|\langle T x, x\rangle|=w(T)$, the numerical radius of $T .($ Recall that $w(T)=\sup \{|\langle T x, x\rangle|:\|x\|=1\}$.$) As before, every$ compact operator attains its numerical radius.

In [3], the following theorem is proved for operators on any uniformly convex space.

THEOREM 6.7. Every operator is a compact perturbation of a numerical-radiusattaining operator. Moreover, the norm of the compact operator can be made arbitrarily small.

$\left({ }^{1}\right)$ Editorial note: See also A. R. Sourour, A short proof of Radjavi's theorem on selfcommutators, Math. Ann. 239 (1979), 137-139, and P. A. Fillmore, C. K. Fong and A. R. Sourour, Real parts of quasinilpotent operators, Proc. Edinburgh Math. Soc. 22 (1979), 263-269. 
Corollary 6.8. (1) Every operator is the sum of two numerical-radius-attaining operators.

(2) Numerical-radius-attaining operators on $H$ are $($ norm) dense in $\mathcal{B}(H)$.

We next consider the class of irreducible operators. An operator $T$ is irreducible if it has no nontrivial reducing subspace. It is well-known that the simple unilateral shift is irreducible [24, Corollary to Problem 147]. The surprising fact, proved by Radjavi [40], is the following

THEOREM 6.9. Every operator is the sum of two irreducible operators.

This is proved as an application of the fact that, for any two nonscalar operators $A_{1}$ and $A_{2}$ there exists an orthonormal basis with respect to which the matrices of $A_{1}$ and $A_{2}$ have only nonzero entries. It improves an earlier result in [14], where the number of irreducible operators required is four.

The last class of operators to be considered is that of cyclic operators. An operator $T$ on $H$ is cyclic if there is a vector $x$ in $H$ such that the closed linear span of $\left\{x, T x, T^{2} x, \ldots\right\}$ is $H$. Very recently, we have been able to show in [52] the abundance of such operators in the additive sense.

THEOREM 6.10. Every operator is the sum of two cyclic operators.

The proof is based on matrix manipulations with operators.

We conclude this paper by a general method due to Fong et al. [15, 17], which is useful in showing that linear combinations of operators from certain classes are equal to the algebra of all operators. One drawback of this method is that we have no control on the number of the special operators in the linear combinations, let alone their minimal numbers. This method is based on the notion of unitarily invariant manifolds of operators.

Let us fix an infinite-dimensional space $H$. A linear manifold $\mathcal{L}$ of operators on $H$ is unitarily invariant if $U^{*} T U \in \mathcal{L}$ for any $T \in \mathcal{L}$ and unitary operator $U$ on $H$. If $\mathcal{L}$ is such a manifold on $H$, then it can be transported to any other (infinite-dimensional) space $K$ : take any unitary transform $W: K \rightarrow H$ and put $\mathcal{L}_{K}=\left\{W^{-1} T W: T \in \mathcal{L}\right\}$. It is easy to see that $\mathcal{L}_{K}$ is also unitarily invariant and its definition does not depend on the particular choice of $W$. Thus, in particular, we may consider $\mathcal{L}_{H \oplus H}$. Let $J_{\mathcal{L}}=\left\{A \in \mathcal{B}(H):\left[\begin{array}{cc}0 & A \\ 0 & 0\end{array}\right] \in \mathcal{L}_{H \oplus H}\right\}$. It can be shown that $J_{\mathcal{L}}$ is an ideal in $\mathcal{B}(H),\left[\begin{array}{ll}A & B \\ C & D\end{array}\right]$ in $\mathcal{L}_{H \oplus H}$ implies $B \in J_{\mathcal{L}}$, and $\left[J_{\mathcal{L}}, \mathcal{B}(H)\right] \subseteq \mathcal{L}$, where $\left[J_{\mathcal{L}}, \mathcal{B}(H)\right]$ denotes the class of linear combinations of commutators formed by operators from $J_{\mathcal{L}}$ and $\mathcal{B}(H)$ (cf. [15, Theorems 1 and 2]). Thus if we know that $J_{\mathcal{L}}=\mathcal{B}(H)$, then we obtain $\mathcal{B}(H)=[\mathcal{B}(H), \mathcal{B}(H)] \subseteq \mathcal{L}$, whence $\mathcal{L}=\mathcal{B}(H)$.

Many of the combination results in the previous sections can be deduced through this method, although now we can say nothing about the number of special operators in such combinations. As an illustration, we prove the following theorem. A weaker version of it was obtained in [45, Corollary 1].

THEOREM 6.11. Let $T$ be an operator on an infinite-dimensional space $H$. Then every operator on $H$ is a linear combination of the operators which are 
unitarily equivalent to $T$ if and only if $T$ is not of the form $\lambda I+K$, where $\lambda$ is a scalar and $K$ is compact.

The necessity is trivial. To prove the sufficiency, let $\mathcal{L}$ be the class of linear combinations of operators unitarily equivalent to $T$. Then $\mathcal{L}$ is obviously unitarily invariant. Note that our assumption on $T$ implies that $T$ is unitarily equivalent to some operator $\left[\begin{array}{cc}A & B \\ C & D\end{array}\right]$ on $H \oplus H$ with $B$ noncompact. Since $\left[\begin{array}{cc}A & B \\ C & D\end{array}\right]$ belongs to $\mathcal{L}_{H \oplus H}$, we have $B \in J_{\mathcal{L}}$. Hence $J_{\mathcal{L}}$, an ideal containing a noncompact operator, must be equal to $\mathcal{B}(H)$ and $\mathcal{L}=\mathcal{B}(H)$ follows.

We remark that there is a finite-dimensional analogue of the preceding theorem. It is a reformulation of the Goldberg-Straus theorem on the "generalized numerical radius" [44].

TheOREM 6.12. Let $T$ be an operator on a finite-dimensional space $H$. Then every operator on $H$ is a linear combination of the operators which are unitarily equivalent to $T$ if and only if $T$ is nonscalar and $\operatorname{tr} T \neq 0$.

So these are all we have for now. Hopefully, they do shed some light on our understanding of the structure of operators in general. Where they will lead us to depends on how significant problems in this area can be discovered and solved and how new links and applications to other areas can be established.

\section{References}

[1] J. Anderson, Commutators of compact operators, J. Reine Angew. Math. 291 (1977), $128-132$.

[2] I. D. Berg, An extension of the Weyl-von Neumann theory to normal operators, Trans. Amer. Math. Soc. 160 (1971), 365-371.

[3] I. D. Berg and B. Sims, Denseness of operators which attain their numerical radius, J. Austral. Math. Soc. 36A (1984), 130-133.

[4] R. Bouldin, The essential minimum modulus, Indiana Univ. Math. J. 30 (1981), 513-517.

[5] A. Brown and C. Pearcy, Structure of commutators of operators, Ann. of Math. 82 (1965), 112-127.

[6] M.-D. Choi and P. Y. Wu, Convex combinations of projections, Linear Algebra Appl. 136 (1990), 25-42.

[7] -, - , Sums of projections, to appear.

[8] Ch. Davis, Separation of two linear subspaces, Acta Sci. Math. (Szeged) 19 (1958), 172187.

[9] P. Fan and C. K. Fong, Which operators are the self-commutators of compact operators?, Proc. Amer. Math. Soc. 80 (1980), 58-60.

[10] - - -, Operators similar to zero diagonal operators, Proc. Roy. Irish Acad. 87A (1988), 147-153.

[11] P. A. Fillmore, Sums of operators with square zero, Acta Sci. Math. (Szeged) 28 (1967), $285-288$.

[12] - On similarity and the diagonal of a matrix, Amer. Math. Monthly 76 (1969), 167-169.

[13] -, On sums of projections, J. Funct. Anal. 4 (1969), 146-152.

[14] P. A. Fillmore and D. M. Topping, Sums of irreducible operators, Proc. Amer. Math. Soc. 20 (1969), 131-133. 
[15] C. K. Fong, C. R. Miers and A. R. Sourour, Lie and Jordan ideals of operators on Hilbert space, ibid. 84 (1982), 516-520.

[16] C. K.Fong and G. J. Murphy, Averages of projections, J. Operator Theory 13 (1985), 219-225.

[17] - - - Ideals and Lie ideals of operators, Acta Sci. Math. (Szeged) 51 (1987), 441-456.

[18] C. K. Fong and A. R. Sourour, Sums and products of quasi-nilpotent operators, Proc. Royal Soc. Edinburgh 99A (1984), 193-200.

[19] C. K. Fong and P. Y. Wu, Diagonal operators: dilation, sum and product, Acta Sci. Math. (Szeged) 57 (1993), 125-138.

[20] U. Haager up, On convex combinations of unitary operators in $C^{*}$-algebras, in: Mappings of Operator Algebras, H. Araki and R. V. Kadison (eds.), Birkhäuser, Boston, 1991, 1-13.

[21] P. R. Halmos, Commutators of operators, Amer. J. Math. 74 (1952), 237-240.

[22] -, Commutators of operators, II, ibid. 76 (1954), 191-198.

[23] —, Two subspaces, Trans. Amer. Math. Soc. 144 (1969), 381-389.

[24] -, A Hilbert Space Problem Book, 2nd ed., Springer, New York, 1982.

[25] R. E. Hartwig and M. S. Putcha, When is a matrix a difference of two idempotents?, Linear Multilinear Algebra 26 (1990), 267-277.

[26] -, -, When is a matrix a sum of idempotents?, ibid. 26 (1990), 279-286.

[27] R. B. Holmes, Best approximation by normal operators, J. Approx. Theory 12 (1974), $412-417$.

[28] S. Izumino, Inequalities on operators with index zero, Math. Japon. 5 (1979), 565-572.

[29] R. V. Kadison and G. K. Pedersen, Means and convex combinations of unitary operators, Math. Scand. 57 (1985), 249-266.

[30] N. J. Kalton, Trace-class operators and commutators, J. Funct. Anal. 86 (1989), 41-74.

[31] J. Lindenstrauss, On operators which attain their norm, Israel J. Math. 1 (1963), 139148.

[32] K. Matsumoto, Self-adjoint operators as a real span of 5 projections, Math. Japon. 29 (1984), 291-294.

[33] Y. Naka mura, Every Hermitian matrix is a linear combination of four projections, Linear Algebra Appl. 61 (1984), 133-139.

[34] K. Nishio, The structure of a real linear combination of two projections, ibid. 66 (1985), 169-176.

[35] C. L. Olsen, Unitary approximation, J. Funct. Anal. 85 (1989), 392-419.

[36] C. L. Olsen and G. K. Pedersen, Convex combinations of unitary operators in von Neumann algebras, ibid. 66 (1986), 365-380.

[37] A. Paszkiewicz, Any self-adjoint operator is a finite linear combination of projections, Bull. Acad. Polon. Sci. Sér. Sci. Math. 28 (1980), 227-245.

[38] C. Pearcy and D. M. Topping, Sums of small numbers of idempotents, Michigan Math. J. 14 (1967), 453-465.

[39] H. Radjavi, Structure of $A^{*} A-A A^{*}$, J. Math. Mech. 16 (1966), 19-26.

[40] - Every operator is the sum of two irreducible ones, Proc. Amer. Math. Soc. 21 (1969), $251-252$.

[41] F. Riesz and B. Sz.-Nagy, Functional Analysis, Frederick Ungar, New York, 1955.

[42] M. Rørdam, Advances in the theory of unitary rank and regular approximation, Ann. of Math. 128 (1988), 153-172.

[43] J. G. Stampfli, Sums of projections, Duke Math. J. 31 (1964), 455-461.

[44] B.-S. Tam, A simple proof of the Goldberg-Straus theorem on numerical radii, Glasgow Math. J. 28 (1986), 139-141.

[45] D. M. Topping, On linear combinations of special operators, J. Algebra 10 (1968), $516-521$. 
[46] J.-H. Wang, Decomposition of operators into quadratic types, Ph.D. dissertation, National Chiao Tung Univ., Hsinchu, Taiwan, 1991.

[47] —, The length problem for idempotent sum, Linear Algebra Appl., to appear.

[48] - Linear combination of idempotents, to appear.

[49] J.-H. Wang and P. Y. Wu, Sums of square-zero operators, Studia Math. 99 (1991), 115-127.

[50] - - - Difference and similarity models of two idempotent operators, Linear Algebra Appl., to appear.

[51] P. Y.W u, Sums of idempotent matrices, ibid. 142 (1990), 43-54.

[52] - Sums and products of cyclic operators, Proc. Amer. Math. Soc., to appear. 